\title{
Helicobacter pylori and Perinatal Pathologies: Pathogen Transmission During Childbirth?
}

\author{
Marcela Espinoza Monje ${ }^{1}$, Ana Marín Vega ${ }^{1}$, Lillian Matamala Valdés ${ }^{2}$ and Apolinaria García Cancino ${ }^{{ }^{\star}}$ \\ ${ }^{1}$ Department of Microbiology, Faculty of Biological Sciences, University of Concepcion, Concepcion, Chile \\ ${ }^{2}$ Department of Obstetrics and Childcare, Faculty of Medicine, University of Concepcion, Concepcion, Chile
}

"Corresponding author: Dr. Apolinaria García Cancino, Department of Microbiology, Faculty of Biological Sciences, University of Concepcion, PO Box 160C, Concepcion, Chile, Tel: 56- 41-2204144/56-41-2204118; E-mail: apgarcia@udec.cl

Received date: June 15, 2016; Accepted date: July 15, 2016; Published date: July 22, 2016

Copyright: (c) 2016 Cancinoa AG, et al. This is an open-access article distributed under the terms of the Creative Commons Attribution License, which permits unrestricted use, distribution and reproduction in any medium, provided the original author and source are credited.

\begin{abstract}
Perinatal pathologies are diseases that show up during pregnancy until after the boy or girl's birth, up to 28 days of life approximately. Helicobacter pylori infection is more frequent during pregnancy because in this period there is a decrease of the immune response mediated by cytotoxic cells with preservation of innate humoral immunity that causes bacterial activation and consequently a negative impact both for the mother's as well as for the fetus's health.

Some of the perinatal pathologies related to this particular infection are: hyperemesis gravidarum, iron-deficiency anemia, restriction of intrauterine growth, spontaneous abortion and preeclampsia.

The purpose of this review is to analyze pathologies linked to Helicobacter pylori infection during the pregnancy and find out if this pathogen can be transmitted from the mother to the child.

It is concluded that the presence of Helicobacter pylori is considered a risk factor for each one of the aforementioned perinatal pathologies. However, this subject still needs further study, altogether with the potential impact of the pre-conception research and Helicobacter pylori infection eradication during this particular period. Vaginal yeast would be the main reservoir of this microorganism and it would enable its transmission to new-born babies.
\end{abstract}

Keywords: Helicobacter pylori; Hyperemesis gravidarum; Irondeficiency anemia; Restriction of intrauterine growth; Spontaneous abortion; Preeclampsia

\section{Introduction}

Helicobacter pylori $(H$. pylori) is a microaerophilic, spiral-shaped bacteria that primarily colonizes the antral gastric mucosa where it causes an acute and chronic inflammation. This infection is also associated with peptic ulcer, adenocarcinoma and gastric lymphoma $[1,2]$. The bacteria is frequently acquired during childhood and it persists throughout lifetime if it is not eradicated. It shows a high incidence level, mainly in under-developed countries. $50 \%$ of the world population is colonized with this particular bacteria. The infection persists throughout lifetime and its spontaneous remission is unusual [3].

H. pylori has several virulence factors that allow epithelial cells colonization; among them urease and flagella are predominant and critical for colonization and pathogenesis, so they are present in all strains. They also have adhesins such as HpaA, BabA, SabA and OipA, for example, that allow its specific adherence to the host's epithelial cells. There are several pathogenicity factors that influence gastric mucosa damage such as vacuolating cytotoxin A (VacA), that causes vacuolisation in epithelial cells, cell death and epithelial integrity destruction; on the other hand, cytotoxin associated to A gene (CagA), coded in a pathogenicity island is associated with severe gastritis, nasal mucosa atrophy, high risk of ulcer and gastric cancer [4]. Seropositivity combined both with CagA and VacA is directly correlated to a high morbidity rate [5].

During the last two decades $H$. pylori has been considered as the responsible factor of many extragastric expressions, that include haematologic pathologies, cardiovascular, diseases, neurological illnesses, metabolic disorders and skin diseases [5]. Possible causal role of $H$. pylori infection in these pathologies is uncertain.

In 1999 Linciers et al. [6] found an increase in IgM levels (marker of recently acquired infection) in pregnant women compared to nonpregnant women, so they suggest that pregnancy itself might increase susceptibility to $H$. pylori's infection. This may be due to the fact that pregnancy is characterized by a decrease of the immune response mediated by cytotoxic cells with preservation of innate humoral immunity [7].

For this reason several studies were started to relate $H$. pylori infection with varied perinatal pathologies (Table 1); perinatal pathologies are defined as all pathologies that appear during pregnancy until after the child is born, approximately until 28 days of life. First ones to be assessed were pathologies related to hyperemesis gravidarum, iron-deficiency anemia, restriction of intrauterine growth (RCIU), spontaneous abortion and preeclampsia (PE) (Figure 1). Another aspect addressed in this revision is to determine if $H$. pylori can actually be transmitted in a vertical way from the mother to the child. 


\begin{tabular}{|l|l|}
\hline Perinatal pathology & Reference \\
\hline Hyperemesis gravidarum & {$[8-12]$} \\
\hline Preeclampsia & {$[13-15,23]$} \\
\hline Iron-deficiency Anemia & {$[16-19]$} \\
\hline Intrauterine growth Restriction & {$[20-22,27]$} \\
\hline Spontaneous abortion & {$[24-26]$} \\
\hline
\end{tabular}

Table 1: Relationship between Helicobacter pylori's infection and perinatal pathologies.

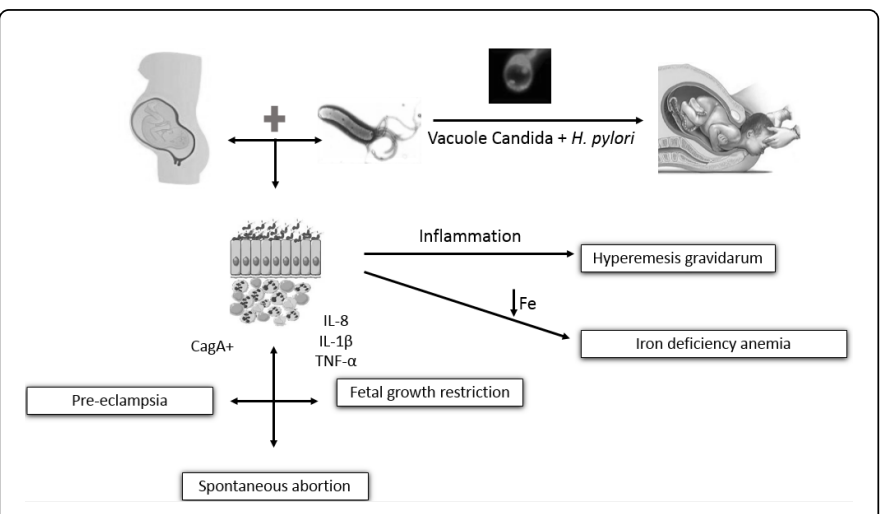

Figure 1: Perinatal pathologies related to Helicobacter pylori infection. H. pylori infection during pregnancy can cause a local and inflammatory damage over the gastric epithelium resulting in gastrointestinal disturbance such as hyperemesis gravidarum. $H$. pylori sequesters essential micronutrients from the host organism such as Iron $(\mathrm{Fe})$ and it causes iron deficiency anemia. In addition, it is noted that the presence of CagA+ strains plus the production of pro-inflammatory cytokines lead to an anti- $H$. pylori antibodies cross reaction that generates a placental lesion that contributes to relevant pathologies during pregnancy, such as Preeclampsia (PE), due to platelet activation, blood clots formation and intrauterine growth restriction (RCIU); in addition, as PE and RCIU are related to an abnormal placentation, this is a relevant factor to trigger a spontaneous abortion. Finally, the existence of intracellular $H$. pylori in Candida's vacuoles present in the vaginal canal of pregnant women has been observed; this is a relevant mode of transmission of the pathogen to the new-born baby.

The purpose of this particular revision is to analyze all pathologies diagnosed during pregnancy that are related to $H$. pylori infection.

The search strategy in PubMed used the following keywords: Helicobacter pylori and perinatal pathologies or pregnancy or hyperemesis gravidarum or preeclampsia or maternal infections or iron-deficiency anemia or intrauterine/fetal growth restriction/ retardation or Spontaneous abortion or Candida albicans reservoir during pregnancy. The bibliographic search covered the time span from 1994 to 2015.

\section{Hyperemesis Gravidarum (HG)}

Nausea and vomiting are the most common symptoms that can affect women during pregnancy with an approximately $80 \%$ prevalence in all pregnancies in different levels; they decrease between 16 to 20 weeks of gestation, but in some cases they persist beyond 20 weeks. In mild cases these symptoms are called emesis gravidarum and they are not considered a pathological situation provided that they don't restrict woman's daily life. Severe cases, called HG, are defined as vomiting of pregnancy and they are serious enough to cause weight loss, dehydration, starvation acidosis, alkalosis due to hydrochloric acid loss and hypokalemia. This particular complication affects $0.3 \%$ to $2.0 \%$ of all pregnancies. The reason to suffer from hyperemesis gravidarum is unknown, but hypotheses include hormonal mechanisms, emotional factors and $H$. pylori infection [8].

Pathogenesis of hyperemesis gravidarum is not quite clear yet. It has been found that when serum level in human chorionic gonadrotrophin (HCG) increases, symptoms of nausea and vomiting show up. When HCG levels in serum decrease, these symptoms disappear. Consequently, most of the researchers believe that high HCG levels in serum are associated to hyperemesis gravidarum. However, it is not easy to prove that HCG levels in serum are not directly proportional to the severity of the symptoms. Another possible etiology is the increase of estrogen levels. This is explained by an increase of liquid accumulation caused by high levels of steroid hormones in pregnant women where a $\mathrm{pH}$ change can happen (this includes gastro-intestinal tract $\mathrm{pH}$ ) and hypothetically it could result in the manifestation of a $H$. pylori sub-clinic infection that may exacerbate gastro-intestinal symptoms.

In Shabana et al. [9] study it was found that there is a strong correlation between $\mathrm{HG}$ and $H$. pylori infection, so they estimate that when a pregnant patient shows evidence of HG symptoms it is necessary to perform a test to confirm $H$. pylori seropositivity.

Güngören et al. [10] carried out a study with pregnant women with HG symptoms; they divided them into three categories, mild, moderate and severe ones; a higher positivity rate of $H$. pylori's DNA by means of PCR was found in 21 pregnant women that corresponded to $87.5 \%$ of the severe category group $(\mathrm{p}=0.001)$. They concluded that there was a positive correlation between $H$. pylori infection and HG. Poveda et al. [11] assessed $H$. pylori in Chilean pregnant women and their link with dyspepsia and HG; they found that after the three first months of gestation these symptoms are significantly correlated with $H$. pylori infection. Li et al. [12] carried out a meta-analysis to validate and strengthen the association between HG and $H$. pylori infection. For this purpose, they searched in varied data bases research studies published until March 20th, 2014. They found that $H$. pylori infection rate was significantly higher in pregnant women with $\mathrm{HG}(\mathrm{p}<0.001)$. In addition, by means of a sub-group analysis, it was found that $H$. pylori infection was also a risk factor in Asia, Oceania and particularly in Africa $(\mathrm{p}<0.001)$; due to these background records it is concluded that H. pylori should be considered as one of the risk factors for HG, especially in under developed countries.

\section{Preeclampsia (PE)}

$\mathrm{PE}$ is defined as the hypertension specifically induced by pregnancy; it is a hypertensive and clotting type of disorder that affects approximately 2-8\% of all pregnancies and it is one of the major causes of maternal and fetal morbidity and mortality [13]. It is characterized by an excessive maternal inflammatory response with high circulating levels of pro-inflammatory cytokines and endothelial injury. 
Page 3 of 5

In spite of the large amount of studies on the subject, little is known about its pathogenic mechanisms; one of the possible hypotheses is that some infectious agents might be a trigger for PE start [13].

In recent years an epidemiological link between $H$. pylori infection and PE has been observed [14].

It has been shown that this particular pathogen enhances platelet activation and blood clot formation; this effect encourages inflammation and endothelial lesion. In consequence, $H$. pylori might cause or directly worsen generalized inflammation and PE's typical endothelial malfunction. In addition, it has been recently observed that $H$. pylori's seropositive individuals suffering from PE are characterized by a more severe inflammatory status and more lipid peroxidation [15].

A stronger link between $\mathrm{PE}$ and infection associated to $H$. pylori's positive Cag-A strains has been found. Recent in vitro-studies have proved that anti-CagA antibodies show a cross reaction with human trophoblastic cells and they determine a functional impairment in terms of cells invasiveness; therefore, the first model of placental damage mediated by a pathogen infection is introduced. However, $H$. pylori infection contribution to PE pathogenesis or to the aggravation of its clinical presentation needs further study as well as the potential impact that $H$. pylori infection might have on the pre-conception stage and the effect of infection eradication in the syndrome incidence [13]. Therefore, these observations provide a possible explanation for the autoimmune pathogenic mechanism induced by $H$. pylori infection which negatively interferes with the development of placentation.

\section{Iron-Deficiency Anemia}

Iron deficiency, defined as low total body iron content is one of the world's most common deficiency. Iron is an essential micro-nutrient necessary for a large variety of biological processes, system immune, cognitive, reproductive functions and the labor performance. During pregnancy, the future mother must deplete her iron stores with the purpose of supply the fetus with the right amount of it [16].

In pregnant women, epidemiological studies suggest that anemia, presumably due to iron deficiency, is related to a higher risk of low birth weight, prematurity and perinatal mortality. In nursing infants and children suffering from iron deficiency there is convincing evidence of damage in their psychomotor development and their cognitive function.

A study carried out by Cárdenas et al. [17] where the key question was if $H$. pylori infection is associated to iron deficiency and anemia caused by iron deficiency in the U.S.A., the conclusion was that $H$. pylori infection is associated to anemia caused by iron deficiency regardless of the presence or absence of peptic ulcer disease.

The biological mechanism by which $H$. pylori causes the alteration in iron stores is not quite understood but it seems to imply several ways including gastro-intestinal blood loss, decrease of iron absorption in the diet and increase of iron absorption by the bacteria [18].

Baingana et al. [19] in a study whose aim is to describe the etiology of anemia en pregnant women in Uganda, analyzes iron deficiency and common infections as contributors to anemia in this particular population. They found that infections and inflammation are more relevant than iron deficiency in the etiology of Uganda's pregnant women during the first quarter of the year.

\section{Intrauterine Growth Restriction (IUGR)}

IUGR is defined as fetus deficient growth while it is in the uterus during gestation without achieving its genetically determined growth potential. IUGR is considered as a severe PE's complication, but it is still not clear if they are both different pathologies or if they are associated to one another. This particular pathology is considered a multifactorial condition where fetal physiopathological, placental and maternal aspects are included, where nutrients reduction, oxygen availability, drug ingestion, decrease of placental mass and flow, congenital infection and chromosomal abnormalities stand out [20].

In Australia in 2002, Eslick et al. [21] observed an association with $H$. pylori infection and low birth weight for the first time and they showed that intra-uterine growth restriction was more common in $H$. pylori seropositive women (13.5\%) compared to seronegative women (6.0\%).

Physiopathological mechanisms by means of which $H$. pylori might affect fetal growth are for the time being speculative; it might be inferred that if $H$. pylori can enhance dyspeptic symptoms, nausea and vomiting during pregnancy [11], it might as well decrease pregnant women's appetite and encourage fetal growth restriction. Another possible explanation is that it can be related to local and systemic inflammatory mechanisms triggered by $H$. pylori, that might influence vascularization and placentation during pregnancy, though they have not been studied well enough during this particular period. A study performed in a murine model suggested that $H$. pylori infection can induce the activation of immune uterine resident cells and/or recruit these cells at an endometrial level, causing an effect on the local Th-1Type response induced by $H$. pylori and change Th1/Th2 systemic balance and cytokins in areas with specific conditions of active tissue and vascular formation, such as pregnancy [22] , bringing about a deficient placental vascularization and in consequence, lesser intrauterine nutrition.

It has recently been proved that anti-CagA antibodies show an in vitro cross reaction on placental tissue that in turn decreases its invasiveness ability [23]; abnormal placentation might lead to a consequent IUGR.

\section{Spontaneous Abortion}

Spontaneous abortion is the spontaneous loss of an embryo or fetus before the 20th week of pregnancy [24]. Most losses are due to chromosomal abnormalities that make fetus development impossible, but there are also other non-chromosomal reasons such as infections. It has already been seen that $H$. pylori infection, as mentioned above, is associated to several pathologies during pregnancy, such as PE and RCIU that are mainly characterized by and abnormal placentation that leads to spontaneous abortion cases. In addition, it has recently been proved that anti-CagA antibodies have a cross reaction with respect to in vitro cytotrophoblast cells and they reduce their invasiveness ability [23]. In a study performed in 2011 by Hajishafiha et al. [25], their inference is that when there is an infection caused by $H$. pylori strains that are CagA positive, there is an increase in the potential of an early abortion (possibly through the increase of inflammatory cytokins release).

Our inference is that $H$. pylori might increase the risk of a spontaneous abortion probability; however, until now there is no direct link between seropositivity with respect to $H$. pylori and recurrent spontaneous abortion [25]. 


\section{Mother-to-child Transmission}

Some researchers consider vertical transmission as a form of acquiring $H$. pylori by newborns from their mothers, during their passage though the birth canal [26]. Up to now there is no report that the bacterium has been isolated directly from the vaginal mucosa but a symbiotic relationship between the yeast Candida albicans and $H$. pylori has been proposed [26].

Several studies demonstrate that bacteria can survive in vacuoles of eukaryotic organisms, such as free living amoebas. These is the case of Enterobacter aerogenes, Aeromonas hydrophila and Legionella pneumophila, which have been observed to survive within vacuoles of Acanthamoeba castellanii, inhibiting the fusion of the phagolysosome [27]. The same phenomenon has been observed for Pseudomonas aeruginosa, growing intracellularly in amoebas of different genera isolated from the pipes of a hospital [28]. This fact called the attention of a group of researchers who asked themselves which eukaryotic might be serving as vehicle and providing protection from the environment to $H$. pylor? They made the relationship between this bacterium and oral yeasts because, as it is known, they can be found as commensals in the mucosa of the oral cavity, intestine and vagina of humans [29].

Siavoshi et al. [26] isolated yeasts from the oral cavity of dyspeptic patients and they were able to amplify, by PCR, $H$. pylori specific genes (16S rRNA and cagA, vacA and ureAB). They also observed, by optic microscopy, the presence of intracellular $H$. pylori located within vacuoles of yeasts belonging to genus Candida. They concluded that possibly $H$. pylori was using Candida as a protector during stressing conditions $[26,30]$. Candida albicans is the yeast species that usually colonizes healthy adults and children as well as hospitalized patients [31]. The most frequently surfaces colonized by Candida albicans are the oral mucosa followed by the vulvovaginal mucosa [31].

Regarding the presence of Candida in the vagina, nearly $40 \%$ of women are asymptomatic Candida carriers [32]. The growth of this microorganism is controlled by the immune response and the presence of other microorganisms, such as Lactobacillus spp. When the equilibrium of the vaginal microbiota is lost and there is an overpopulation of Candida, a vulvovaginal candidiasis (VVC) is produced [33].

VVC is a rather common in adult women, showing its highest incidence between the ages ranging from 20 to 40 years. It is estimated that $50 \%$ of all women will have at least one VVC episode by the time they become 25 years old [34,35] and that among pre-menopausal women $75 \%$ of them will suffer at least one VVC episode while $45 \%$ of them will suffer two or more episodes [36]. Candida albicans, a commensal microorganism which can be isolated from the vagina, is the species responsible for most VVC cases. VVC can be also caused by other Candida species but their frequency is much lower

During pregnancy, the vagina is more predisposed to infections. The literature reports that colonization by Candida spp. is always higher in pregnant women when compared to non-pregnant females. According to a recent report, up to $28 \%$ of pregnant women can have Candida spp. in their vaginal discharge [37]. Estrogen level increase causing increased glycogen content in the vagina, increased adhesiveness of epithelial vaginal cells, and strengthening of the mycelia formation by the presence of estrogen specific receptors the cytosol of yeasts have been described as pathogenic mechanisms. Furthermore, a reduced local immunity also facilitates vaginal colonization and subsequent infection [31].
Colonization of newborns by Candida albicans is an early event occurring by vertical transmission during the transit through the birth canal and acquired by oral ingestion of yeasts [38-40].

Considering the background information, Siavoshi et al. [26] asked themselves if vaginal yeasts of pregnant women could also contain $H$. pylori. Therefore, they analyzed 108 vaginal samples obtained from pregnant women and found that 30 of them (27.8\%) contained Candida spp. and $18 / 30(60 \%)$ were positive for $H$. pylori. They also detected $H$. pylori genes in $5 / 7$ yeasts isolated from the oral cavity of newborns from the mothers considered in their study, showing concordance between the genotype of $H$. pylori found intracellularly in Candida spp. and H. pylori found in Candida spp. in the oral cultures of newborns. They concluded that another possible way of early transmission of $H$. pylori infection could be the acquisition of this bacterium located intracellularly in Candida spp. during the passage through the birth canal; i.e., by vertical transmission [41].

\section{Conclusion}

H. pylori at present is not only studied in association to gastrointestinal disorders during pregnancy, but also in connection to other severe disturbances that are developed during this particular period. Each one of the pathologies introduced in this revision lead us to recognize various mechanisms by means of which $H$. pylori might have some influence; these include competition for micro-nutrients, induction of inflammatory mediators and cross reaction between antiH. pylori antibodies and antigens existing in trophoblastic cells. Moreover, these mechanisms and the various pathologies are closely interrelated, so that $H$. pyloris presence is considered a risk factor for each one of them as a whole; thus, iron deficiency anemia might indirectly be the consequence of the local and systemic inflammation induced by the infection of this particular pathogen.

A relevant aspect worth mentioning is that until recently $H$. pylori transmission from the mother to the child did not seem to happen during birth. However, intracellular existence of $H$. pylori in Candida yeast vacuoles has been proved; this might act as a bacteria reservoir and play a relevant role in its transmission to a new host.

Finally, more data are necessary to assess the actual meaning of studies carried out up to now, because they are still limited with respect to their ability to evaluate these kinds of pathologies.

\section{Conflict of Interest:}

The authors report no conflicts of interest.

\section{References}

1. Herrera V, Parsonnet J (2009) Helicobacter pylori and gastric adenocarcinoma. Clin Microbiol Infect 15: 971-6.

2. Marshall B, Windsor H (2005) The relation of Helicobacter pylori to gastric adenocarcinoma and lymphoma: pathophysiology, epidemiology, screening, clinical. Med Clin North Am 89: 313-44.

3. Kim S, Ruiz V, Carroll J, Moss S (2011) Helicobacter pylori in the pathogenesis of gastric cancer and gastric lymphoma. Cancer Lett 305: 228-38.

4. Chromvarin C, Namwat W, Chaicumpar K, Mairiang P, Sangchan A, et al. (2008) Prevalence of Helicobacter pylori vacA, cagA, cagE, iceA and babA2 genotypes in Thai dyspeptic patients. Int J Infect Dis 1: 30-6.

5. Tan H, Goh K (2012) Extragastrointestinal manifestations of Helicobacter pylori infection: facts or myth? A critical review. J Dig Dis 13: 342-9. 
6. Lanciers S, Despinasse B, Mehta D, Blecker U (1999) Increased susceptibility to Helicobacter pylori infection in pregnancy. Infect Dis Obstet Gynecol 7: 195-8.

7. Chang J, Streitman D (2012) Physiologic adaptations to pregnancy. Neurologic Clinics 30: 781-789.

8. Mansour G, Nashaat E (2009) Helicobacter pylori and hyperemesis gravidarum. Int J Gynaecol Obstet 106: 63-4.

9. Güngören A, Bayramoğlu N, Duran N, Kurul M (2013) Association of Helicobacter pylori positivity with the symptoms in patients with hyperemesis gravidarum. Arch Gynecol Obstet 288: 1279-83.

10. Poveda GF, Carrillo KS, Monje ME, Cruz CA, Cancino AG (2014) Helicobacter pylori infection and gastrointestinal symptoms on Chilean pregnant women. Rev Assoc Med Bras 60: 306-10.

11. Jacobson G, Autry A, Somer-Shely T, Pieper K, Kirby R (2003) Helicobacter pylori seropositivity and hyperemesis gravidarum. Journal of Reproductive Medicine 48: 578-82.

12. Li L, Li L, Zhou X, Xiao S, Gu H, et al. (2015) Helicobacter pylori Infection Is Associated with an Increased Risk of Hyperemesis Gravidarum: A Meta-Analysis. Gastroenterology Research and Practice 278905.

13. Tersigni C, Franceschi F, Todros T, Cardaropoli S, Scambia G, et al. (2014) Insights into the Role of Helicobacter pylori Infection in Preeclampsia: From the Bench to the Bedside. Front Immunol 5: 484.

14. Ponzetto A, Cardaropoli S, Piccoli E, Rolfo A, Gennero L, et al. (2006) Pre-eclampsia is associated with Helicobacter pylori seropositivity in Italy. J Hypertens 24: 2445-9.

15. Cardaropoli S, Rolfo A, Piazzese A, Ponzetto A, Todros T (2011) Helicobacter pylori's virulence and infection persistence define preeclampsia complicated by fetal growth retardation. World Journal of Gastroenterology 17: 5156-65.

16. McArdle H, Gambling L, Kennedy C (2014) Iron deficiency during pregnancy: the consequences for placental function and fetal outcome. Proc Nutr Soc 73: 9-15.

17. Cárdenas V, Mull Z, Ortiz M, Graham D (2008) Iron Deficiency and Helicobacter pylori Infection in the United States. Am J Epidemiol 163: 127-34.

18. Muhsen K, Cohen D (2008) Helicobacter pylori infection and iron stores: a systematic review and meta-analysis. Helicobacter 13: 323-40.

19. Baingana R, Enyaru J, Tjalsma H, Swinkels D, Davidsson L (2014) The aetiology of anaemia during pregnancy: a study to evaluate the contribution of iron deficiency and common infections in pregnant Ugandan women. Public Health Nutrition 15: 1-13.

20. Cetin I, Foidart J, Miozzo M, Raun T, Jansson T, et al. (2004) Fetal growth restriction: a workshop report. Placenta 25: 753-57.

21. Eslick G, Yan P, Xia H, Murray H, Spurrett B, et al. (2002) Foetal intrauterine growth restrictions with Helicobacter pylori infection. Alimentary Pharmacology \& Therapeutics 16: 1677-82.

22. Rossi G, Romagnoli S, Lauretti L, Pancotto L, Taccini E, et al. (2004) Helicobacter pylori Infection Negatively Influences Pregnancy Outcome in a Mouse Model. Helicobacter 9: 152-7.

23. Franceschi F, Di Simone N, D’Ippolito S, Castellani R, Di Nicuolo F, et al. (2012) Antibodies anti-CagA cross-react with trophoblast cells: a risk factor for pre-eclampsia?. Helicobacter 17: 426-34.
24. Brown S (2008) Miscarriage and its associations. Seminars in Reproductive Medicine 26: 391-400.

25. Hajishafiha M, Ghasemi-Rad M, Memari A, Naji S, Mladkova N, et al. (2011) Effect of Helicobacter pylori infection on pregnancy rates and early pregnancy loss after intracytoplasmic sperm injection. International Journal of Women's Health 3: 329-35.

26. Siavoshi F, Taghikhani A, Malekzadeh R, Sarrafnejad A, Kashanian M, et al. (2013) The role of mother's oral and vaginal yeasts in transmission of Helicobacter pylori to neonates. Archives of Iranian medicine 16: 288-94.

27. Rudnicka K, Graczykowski M, Tenderenda M, Chmiela M. (2014) Helicobacter pylori morphological forms and their potential role in the transmission of infection. Postẹpy higieny i medycyny doświadczalnej 68: 219-29.

28. Bozue JA, Johnson W (1996) Interaction of Legionella pneumophila with Acanthamoeba castellanii: uptake by coiling phagocytosis and inhibition of phagosome-lysosome fusion. Infection and immunity 64: 668-73.

29. Michel R, Burghardt H, Bergmann H (1995) Acanthamoeba, naturally intracellularly infected with Pseudomonas aeruginosa, after their isolation from a microbiologically contaminated drinking water system in a hospital. International journal of hygiene and environmental medicine 196: 532-44.

30. Siavoshi F, Salmanian AH, Akbari F, Malekzadeh R, Massarrat S (2005) Detection of Helicobacter pylori-specific genes in the oral yeast. Helicobacter 10: 318-22.

31. Cararach M, Comino R, Davi E, Marimo E, Martínez J, et al. (2013) La vulvovaginitis candidiásica recurrente. Progresos de Obstetricia y Ginecología 56: 108-16.

32. Goncalves B, Ferreira C, Alves CT, Henriques M, Azeredo J, et al. (2015) Vulvovaginal candidiasis: Epidemiology, microbiology and risk factors. Crit Rev Microbiol 21: 1-23.

33. Priestley CJ, Jones BM, Dhar J, Goodwin L (1997) What is normal vaginal flora? Genitourinary Medicine 73: 23-8.

34. Geiger AM, Foxman B (1995) The epidemiology of vulvovaginal candidiasis among university students. Am J Public Health 85: 1146-8.

35. Geiger AM, Foxman B (1996) Risk factors for vulvovaginal candidiasis: a case-control study among university students. Epidemiology 7: 182-7.

36. Harley R, De Louvois J (1979) Candida vaginitis. Postgraduate Medical Journal 55: 645-7.

37. García Heredia García SD, Copolillo ES, Cora Eliseth M, Barata MD, et al. (2006) Prevalencia de candidiasis vaginal en embarazadas. Identificación de levaduras y sensibilidad a antifúngicos. Revista Argentina de Microbiología 38: 9-12.

38. Odds FC (1988) Candida and candidosis. (2ndedn), Baillie're Tindall, London.

39. Dignani MC, Solomkin JS, Anaissie E (2003) Candida. In: Anaissie E, McGinnis MR, Pfaller MA (eds.) Medical Mycology. Churchill Livingstone, Filadélfia, pp. 195-239

40. De Beule K, Van Gestel J (2001) Pharmacology of Itraconazol. Drugs 61: 27-37.

41. Salmanian AH, Siavoshi F, Beyrami Z, Latifi-Navid S, Tavakolian A, et al. (2012) Foodborne Yeasts Serve As Reservoirs Of Helicobacter pylori. Journal of Food Safety 32: 152-60. 